\title{
Trapped-Particle-Mediated Damping and Transport
}

\author{
C. Fred Driscoll*, Andrey A. Kabantsev*, Terance J. Hilsabeck* and \\ Thomas M. O'Neil*
}

${ }^{*}$ Dept. of Physics, University of California at San Diego, La Jolla CA USA 92093-0319

\begin{abstract}
Weak axial variations in $B(z)$ or $\phi(z)$ in Penning-Malmberg traps cause some particles to be trapped locally. This causes a velocity-space separatrix between trapped and passing populations, and collisional separatrix diffusion then causes mode damping and asymmetry-induced transport. This separatrix dissipation scales with collisionality as $v^{1 / 2}$, so it dominates in low collisionallity plasmas. The confinement lifetime in the "CamV" apparatus was dominated by a weak magnetic ripple with $\delta B / B \sim 10^{-3}$, and it appears likely that the ubiquitous $(L / B)^{-2}$ lifetime scalings and other applied asymmetry scalings represent similar TPM effects. TPM transport will limit the containment of large numbers of positrons or $\bar{p} \mathrm{~s}$, since TPM loss rates generally scale as total charge $Q^{2}$, independent of length.
\end{abstract}

\section{INTRODUCTION}

Two years ago, "trapped particle asymmetry" modes were reported to occur when an applied "squeeze" voltage causes some particles to be trapped axially; and a simple theory explained the observed mode frequencies [1]. Now, it appears likely that trappedparticle-mediated (TPM) effects are dominant in plasma lifetime scalings, in transport from applied asymmetries, and in diocotron mode damping. This talk will give an overview of what is known $[2,3,4,5]$, where more experiments are needed, and where the theory is lacking.

Electric or magnetic trapping probably occurs in all "long" apparatuses: unintended wall potential variations of 0.1 Volts are common, and it is sobering to note that $\delta B / B=$ $10^{-3}$ will trap $3 \%$ of the particles. Initial experiments (and all theory to date) considered electric trapping; but magnetic trapping is probably more common and important.

Early experiments focused on the new modes (now called "trapped particle diocotron" modes); but the important effect is particles scattering across the trapping separatrix. This breaks the $\mathrm{v}_{z}$ adiabatic invariant, allowing $2 \mathrm{D}$ potential energy to flow to $3 \mathrm{D}$ kinetics, and enabling external asymmetries to generate strong transport. The effect is dominant in low-collisionality plasmas because this separatrix dissipation scales with collisionality as $v^{1 / 2}$, whereas most other effects scale as $v^{1}$. Here, the collisionality can be electron-electron, electron-neutral, or externally stimulated. The effect can be also be described as dissipation of asymmetry-induced equilibrium currents, as in the analysis of bootstrap current in Tokamaks. 


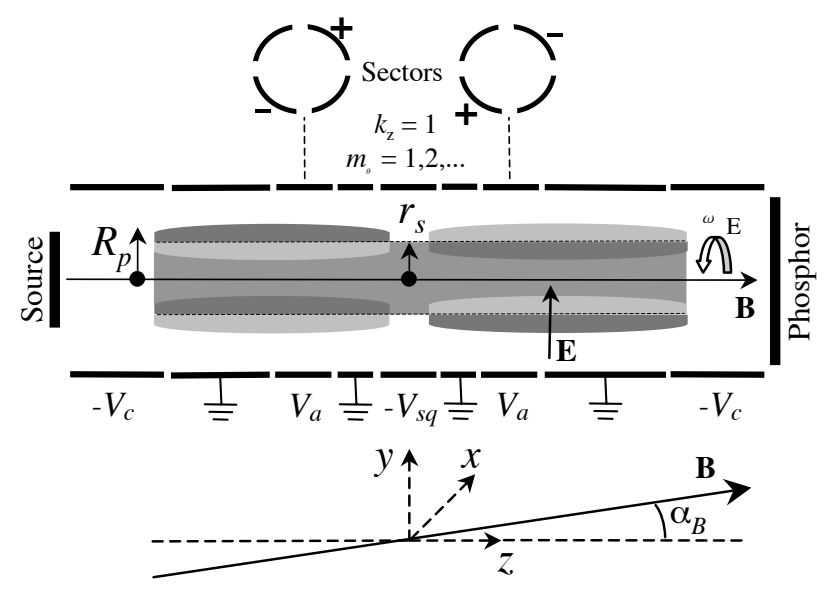

FIGURE 1. Schematic of electron plasma with a trapped particle mode in the cylindrical containment system.

Thus, it now appears likely that most of the $(L / B)^{-2}$ lifetime scalings from "background asymmetries" [6] can be given interpretation in terms of the (partially) known scalings for TPM transport. The measurements of transport from applied electric and magnetic asymmetries [7, 8, 9, 10] also should be compared to TPM predictions. "Anomalous" damping of diocotron modes $[7,11,12]$ is almost certainly related to TPM effects, since TPM damping scales as $B^{-3}$. TPM transport also has important implications for containment of large numbers of positrons or pBars, since the TPM loss rate for magnetic asymmetries scales as total charge $Q^{2}$, independent of length.

Theory provides a reasonable picture of trapped-particle-mode damping with electric trapping [5], but modes in the magnetic trapping case remain enigmatic. Theory can not yet explain the observed particle transport scalings for either case, but this appears imminent for electric trapping. Diocotron mode damping has not been worked out theoretically.

\section{ELECTRIC TRAPPING: NEW MODE}

The experiments are performed on magnetized pure electron plasmas confined in the cylindrical "CamV" apparatus, as shown in Fig. 1. The electron plasmas have density $n \sim 10^{7} \mathrm{~cm}^{-3}$, length $L_{p} \sim 40 \mathrm{~cm}$, radius $R_{p} \sim 1.5 \mathrm{~cm}$, and temperature $T \sim 1 \mathrm{eV}$.

Controlled electric trapping from an applied central "squeeze" voltage $-V_{\mathrm{sq}}$ causes electrons with axial velocity less than the separatrix velocity to be trapped in one end or the other; here, $\mathrm{v}_{s}$ is defined by $\mathrm{v}_{s}^{2}(r) \equiv \frac{2 e}{m} V_{\mathrm{sq}}(r)$. For small $V_{\mathrm{sq}}$, a fraction $N_{L}^{(\mathrm{tr})} / N_{L} \sim 1.2\left(V_{\mathrm{sq}} / \phi_{p}\right)$ of the electrons are trapped, predominantly at $r \sim R_{p}$; here, $N_{L} \equiv \int 2 \pi r d r n$.

This trapping enables novel "trapped particle diocotron modes" with various $m_{\theta}=$ $1,2, \ldots$; but we focus here on $m_{\theta}=1$. The mode frequency $f_{a}$ ranges from the edge rotation frequency $f_{E}\left(R_{p}\right) \sim(100 \mathrm{kHz}) B[\mathrm{kG}]^{-1}$ at low $V_{\mathrm{sq}}$, down to the $k_{z}=0$ diocotron 


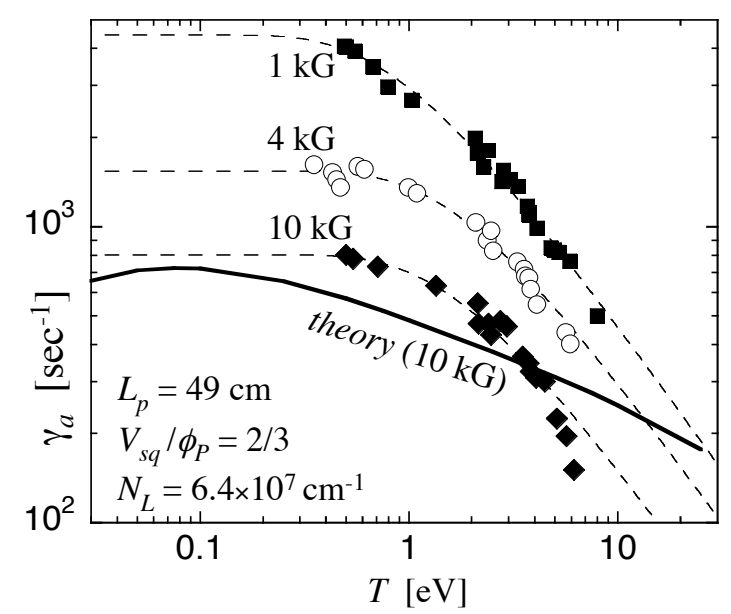

FIGURE 2. Measured damping rate $\gamma_{a}$ versus temperature for 3 magnetic fields; with theory prediction for 1 field.

mode frequency $f_{d}$ at $V_{\mathrm{sq}} \gtrsim \phi_{p}$. The modes are anti-symmetric in $z$, with trapped particles on either end executing $\mathbf{E} \times \mathbf{B}$ drift oscillations that are $180^{\circ}$ out of phase, while passing particles move along field lines in response to the potential from the trapped particles.

At large $V_{\mathrm{sq}}$, the plasma is essentially cut in half, and on either side of the barrier the plasma supports $k_{z}=0$ (diocotron) drift orbits which are $180^{\circ}$ out of phase. Even for small $V_{\mathrm{sq}}$, the trapped particle mode is essentially uniform with $z$ on either side of the barrier, changing sign at the barrier. A simple kinetic theory model with a zero-length trapping barrier [1] predicts mode frequencies agreeing with measurements to within about $10 \%$.

This trapped particle mode is readily excited by $m_{\theta}=1 z$-antisymmetric voltages, as shown in Fig. 1. The excited mode then rings down exponentially, and the damping rate $\gamma_{a}$ is unambiguously obtained. Figure 2 shows the measured $\gamma_{a}$ for three magnetic fields as the plasma temperature is varied; the dash lines represent the generic functional form $a[1-\exp (-b / T)]$. The modes are strongly damped at low temperatures, but the damping decreases precipitously as $T$ increases.

Theory analysis of damping from collisional scattering across the trapping separatrix gives factor-of-two agreement with experiments; but significant discrepancies remain. Since particles on either side of the separatrix are involved in completely different types of motion, there is a discontinuity in the perturbed particle distribution function. As a result, electron-electron collisions produce a large flux of particles across the separatrix. The continual trapping and detrapping of particles results in radial transport of particles and in mode damping, and is readily observed in computer simulations [13].

These collisions at rate $v$ have been treated by a Fokker-Planck collision operator [5], in an analysis similar to that used for the dissipative trapped-ion instability by Rosenbluth, Ross, and Kostomarov [14]. Velocity space diffusion acting for one mode period smoothes out the separatrix discontinuity over a width $\delta \mathrm{v}_{t} \approx \overline{\mathrm{v}} \sqrt{v / f_{E}}$, and the 
damping includes this dependence. The predicted damping rate can be expressed as

$$
\gamma_{a}=\frac{\frac{2 \sqrt{\pi} B}{m_{\theta}} \int_{0}^{R_{w}} r d r|\delta \phi| \overline{\mathrm{v}} \sqrt{\frac{v}{f_{E}^{*}}}\left[\frac{2 \pi e f_{M}}{T}-\frac{c}{B r} \frac{\partial f_{M}}{\partial r} \frac{m_{\theta}}{f_{E}^{*}}\right]_{\mathrm{v}=\mathrm{v}_{s}}}{\int_{0}^{R_{w}} d r \frac{|\delta \phi|^{2}}{f_{*}^{2}} \frac{\partial n_{t}}{\partial r}}
$$

where $\phi(r)$ is the mode potential, $f_{E}^{*}(r) \equiv m_{\theta} f_{E}(r)-f_{a}, n_{t}$ is the trapped density, and $f_{M}$ is the Maxwellian distribution.

Figure 2 shows that this predicts a somewhat less abrupt temperature dependence than is actually observed. This may be related to another significant discrepancy: experiments show non-zero $\left(\sim 20^{\circ}\right)$ phase shifts in the mode eigenfunction $\phi(r)$, whereas no shifts are predicted. The square root provides a significant enhancement, since $v / f_{E}$ is small. The damping rate is expected to have a strong and complicated temperature dependence through the density of particles at the separatrix velocity $\mathrm{v}_{S}(r)$, through the collisional frequency $v$, and through the Debye shielding length $\lambda_{D}$.

\section{ELECTRIC TRAPPING AND TILT: TRANSPORT}

When $\theta$-asymmetries exist in the electric or magnetic confinement fields, they create torques which change the canonical angular momentum $P_{\theta}$ of the plasma, causing the plasma radius to vary. These asymmetry-induced torques are stronger when the symmetric squeeze trapping is present. If the asymmetry is not static, the sign of the torque can be positive or negative. The "rotating wall" confinement technique utilizes wall voltages rotating faster than $f_{E}$ to obtain plasma compression $[15,16]$. For the present experiments, the $\theta$-asymmetries are static in the lab frame and exert a negative torque on the electrons, resulting in bulk radial expansion.

Here, we focus on the $m_{\theta}=1, k_{z}=1$ asymmetry induced by a magnetic tilt, with $B=B\left(\hat{z}+\alpha_{B x} \hat{x}+\alpha_{B y} \hat{y}\right)$; or by the electric "tilt" induced by static $m_{\theta}=1$ voltages $V_{a}$ applied antisymmetrically in $z$ (Fig. 1). The asymmetry-induced transport rate is defined by the rate of plasma expansion

$$
v_{p} \equiv \frac{1}{\left\langle r^{2}\right\rangle} \frac{d\left\langle r^{2}\right\rangle}{d t} \approx \frac{-1}{P_{\theta}} \frac{d P_{\theta}}{d t} .
$$

The expansion rate is found to be proportional to the tilt angle $\alpha_{B}^{2}$, as shown in Fig. 3 . Here, $v_{p}\left(\alpha_{B x}\right)$ is quadratic about $\alpha_{B x}=0$, but the minimum of $v_{p}\left(\alpha_{B y}\right)$ is offset by the separate electric tilt $\alpha_{E y}$ from an applied $V_{a y}$. Indeed, electric and magnetic tilts add vectorially when the proper $z$-averaged electrostatic offsets [17] are calculated, as

$$
\alpha_{E} \equiv(0.51)\left(\frac{4 R_{w}}{L_{p}}\right)\left(\frac{V_{a}}{e N_{L}}\right)\left(\frac{L_{a}}{L_{p}}\right) .
$$

Here, $V_{a}$ is applied to sectors of length $L_{a}$, and the factor 0.51 represents the $m_{\theta}=1$ Fourier coefficient for the (four) $25^{\circ}$ sectors used. The deviation from this quadratic scaling at larger $v_{p}$ (dashed line) is due to an increase of the plasma temperature caused by fast radial expansion. 


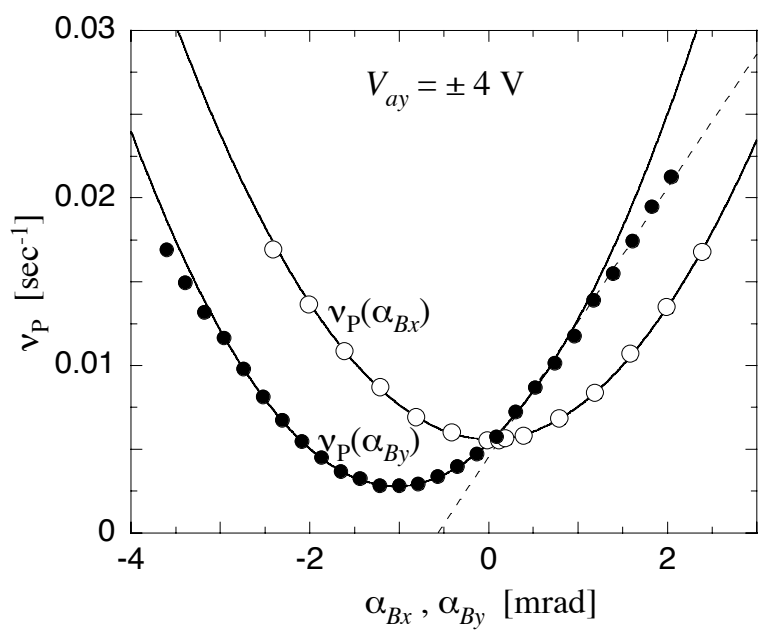

FIGURE 3. Measured transport rate $v_{p}$ from a magnetic tilt with simultaneously applied electric symmetry.

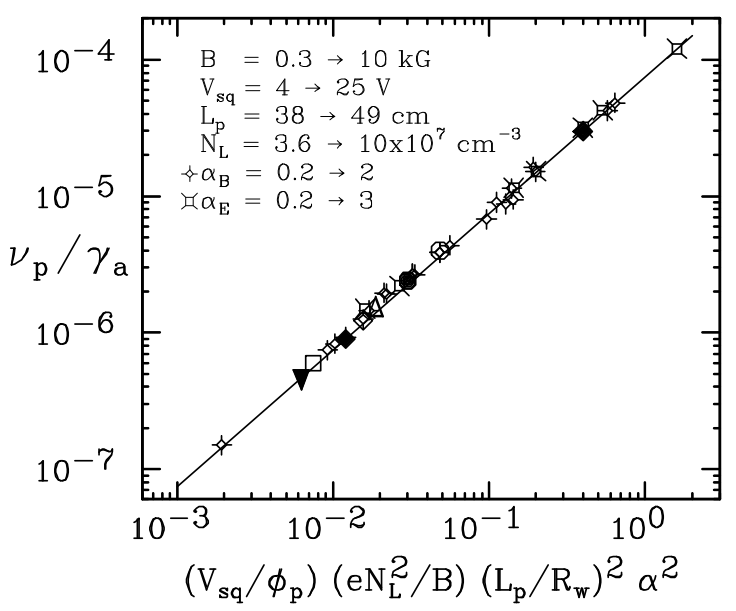

FIGURE 4. Measured normalized transport rate $v_{p} / \gamma_{a}$ vs scalings for all plasma parameters.

The expansion rate $v_{p}$ shows rather complicated dependencies on plasma parameters $n, T, R_{p}, L_{p}$ and $B$, unless the ratio $v_{p} / \gamma_{a}$ is considered. Essentially, we find that all the complicated dynamics is in the separatrix dissipation which causes $\gamma_{a}$. Measuring $\gamma_{a}$ coincident with $v_{p}$ (by exciting the trapped particle mode and watching it decay) allows us to accurately obtain the ratio $v_{p} / \gamma_{a}$.

Figure 4 shows that the ratio $v_{p} / \gamma_{a}$ has only simple power-law dependencies on plasma parameters, as $L_{p}^{2}, B^{-1}$, and $N_{L}^{2}$, where $N_{L} \sim \pi R_{p}^{2} n$. We note that all temperature dependence is in $\gamma_{a}$, that $V_{\mathrm{sq}}$ is normalized to the plasma potential $\phi_{p}$ at $r=0$, and that $\phi_{p} \propto N_{L}$.

With the applied electric trapping and the applied tilt, a single trapped-particlemediated damping and transport process is dominant; and this process exhibits stunningly simple and accurate scalings over 3 decades in $v_{p} / \gamma_{a}$, representing 4 decades in 


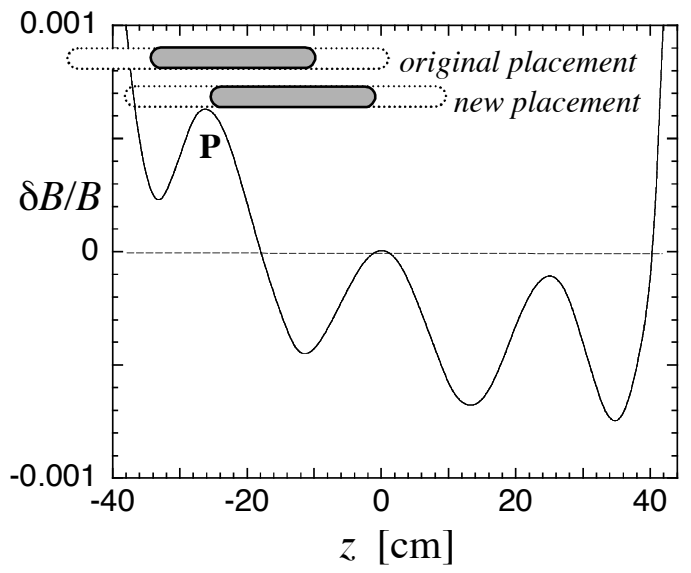

FIGURE 5. Modified electrode placement relative to magnetic ripples exhibits $5 \times$ less background transport.

$v_{p}$. However, the theory of this transport scaling is still incomplete.

\section{MAGNETIC TRAPPING AND TILT: TRANSPORT BUT NO MODE}

Applying a central magnetic "squeeze" $B(z)$ instead of the electric squeeze also causes particle trapping in either end, and causes enhanced transport from electric or magnetic tilts. However, experiments have not yet identified a corresponding trapped particle mode. Presumably, this is because the magnetic trapped particle "mode" has $f_{a}^{(M)}=0$, or $\gamma_{a}^{(M)} / f_{a}^{(M)} \sim 1$. This eliminates the conceptual advantage of relating $v_{p}$ to $\gamma_{a}$; but experiments demonstrate conclusively that scatterings across the magnetic separatrix produce transport, as in the electric trapping case.

These magnetic trapping effects have been studied using an axially centered coil which generates a magnetic mirror of strength $\beta \equiv(\delta B / B) \lesssim 4 \%$ at $B=1 \mathrm{kG}$; and also using the ripples of strength $\beta \approx 10^{-3}$ inherent in our superconducting solenoid.

Surprisingly, these magnetic ripples with $\delta B / B \sim 10^{-3}$ are sufficient for TPM transport to dominate the "background losses." Figure 5 plots the vendor-calculated ripples in the CamV superconducting magnet, together with two axial placements of the electrode stack (shown dotted). In the original placement, the magnetic mirror $P$ occurred within the electron containment region (shown grey). Moving the electrodes by $+9 \mathrm{~cm}$ moved the peaks to the ends of the plasma, and reduced the background transport by $5 \times$. This, together with more subtle probes described below, conclusively establishes these weak mirrors as generators of asymmetry-induced transport.

One expects particles with small pitch angle to be trapped, i.e. those with $\mathrm{v}_{z}<$ $\beta^{1 / 2} \mathrm{v}_{\perp}$. The fraction of these trapped particles is expected to scale as $\beta^{1 / 2}$, giving $0.03 \lesssim N_{L}^{(\operatorname{tr})} / N_{L} \lesssim 0.2$. Moreover, there are theoretical and experimental reasons [18] to expect that the mirror field causes the electrostatic potential to vary along a field line 


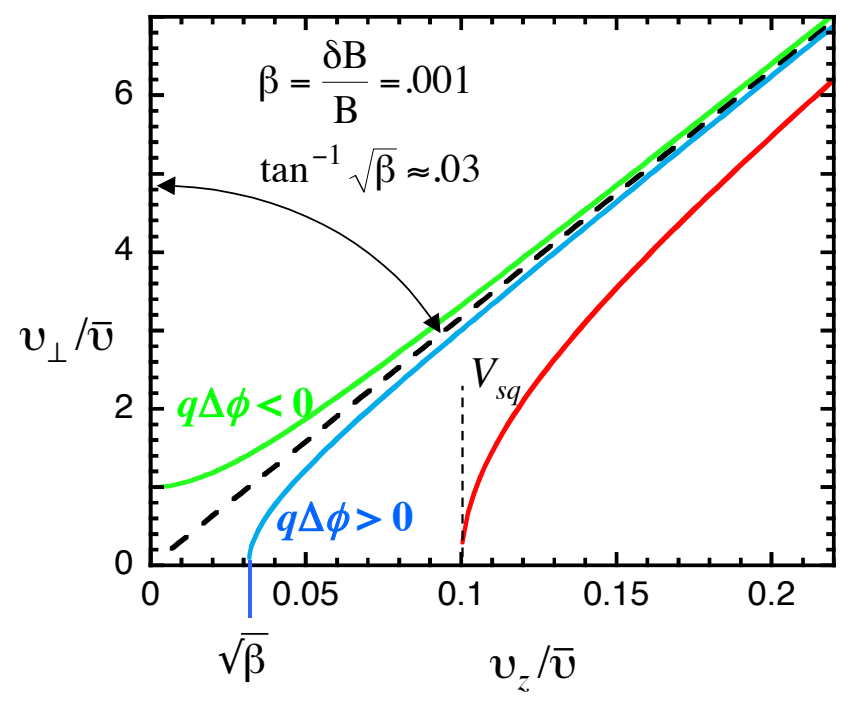

FIGURE 6. The magnetic separatrix with potentials.

by $\Delta \phi$; and an applied electric squeeze voltage $V_{\text {sq }}$ would add in analogously. Thus, the magnetic/electric separatrix is given by

$$
\mathrm{v}_{z}^{2}=\left(\frac{\delta B}{B}\right) \mathrm{v}_{\perp}^{2}+\frac{-2 e}{m}\left[\Delta \phi+V_{\mathrm{sq}}(r)\right]
$$

These hyperbolic separatrices are shown in Fig. 6 for $\beta=10^{-3}$, showing the $\beta^{1 / 2}$ reduction in relevant $\mathrm{v}_{z}$ velocities. The lack of radial separation between trapped and untrapped particles makes $f_{a}^{(M)}=0$ plausible, since the charge separation and $\mathbf{E} \times \mathbf{B}$ drifts characterizing the electric mode may not occur. These trapped particles can be directly detected by selective dumping techniques, but the relevant parallel velocities are substantially less than $\bar{v}$, so measurements to date are only qualitative.

More incisively, the separatrix can be mapped out by enhancing $\mathrm{v}_{z}$ separatrix scatterings with a resonant RF field. Figure 7 shows the transport enhancement when a RF wiggle is applied near a magnetic minimum. Here, we have utilized the "sheath transport" resonance to interact with particles with small $\mathrm{v}_{z}$ : electrons receive a nonadiabatic kick if they have $\mathrm{v}_{z} \approx L^{*} f_{\mathrm{RF}}$, where $L^{*} \equiv 2 R_{w} / j_{01}$ is the axial extent of the wiggle electric fields. The transport response peak in Fig. 7 is as expected for a Maxwellian distribution of particles along the naive $(\Delta \phi=0)$ magnetic separatrix; surprisingly, recent calculations show that $\Delta \phi$ does not affect this resonance curve [18].

Alternately, adding an electric squeeze at the $z$-position of a magnetic mirror moves the separatrix so as to exclude particles with small $\mathrm{v}_{z}$. This causes a reduction in $v_{p}$ for small $V_{\mathrm{sq}}$, as $\mathrm{v}_{z} \lesssim \overline{\mathrm{v}}$ particles are excluded from the magnetic separatrix; and causes an increase in $v_{p}$ for large $V_{\mathrm{sq}}$ as the radially localized electric separatrix becomes dominant. These experimental probes of the separatrix are all quantitatively consistent. 


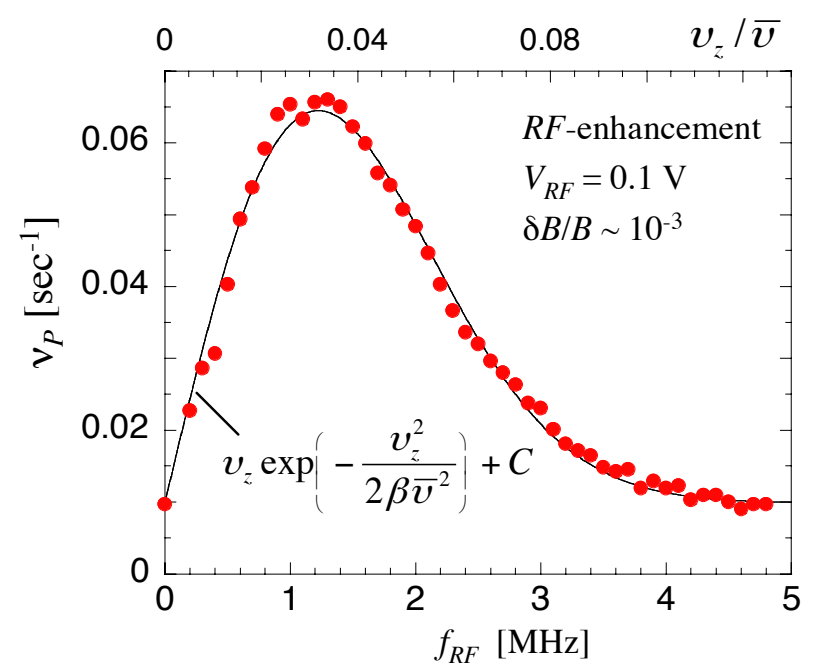

FIGURE 7. RF wiggle causes resonant $\mathrm{v}_{z}$ separatrix-crossings and enhanced transport.

\section{COMMON CHARACTERISTICS: SEPARATRIX DISSIPATION}

The magnetic TPM effects appear to be analogous to the electric TPM effects, except for the radial localization of the electric separatrix and the absence of a detectable magnetic mode. For electric trapping with $V_{\mathrm{sq}}$ acting on magnetic tilt asymmetry $\alpha$, we find

$$
v_{p}^{(E)} \propto L_{p}^{2} B^{-(1.5 \rightarrow 2)} N_{L}^{1 \rightarrow 2} T^{-?} V_{\mathrm{sq}}^{?} \alpha^{2}
$$

where ? represents non-power-law scalings. Most of the complication lies in the separatrix dissipation process, and measurement of $\gamma_{a}$ allows this to be written

$$
\frac{v_{p}^{(E)}}{\gamma_{a}^{(E)}}=\left(6.3 \times 10^{-5}\right)\left(\frac{L_{p}}{R_{w}}\right)^{2}\left(\frac{e N_{L}^{2}}{B}\right)^{1}\left(\frac{N_{L}^{\mathrm{tr}}}{N_{L}}\right)^{1} \alpha^{2} ;
$$

this is valid for both magnetic and electric tilts. For magnetic trapping from $\delta B / B$ acting on tilt $\alpha$ we find

$$
v_{p}^{(M)} \propto L_{p}^{2} B^{-(1.5 \rightarrow 2)} e N_{L}^{2} T^{-?}\left(\frac{\delta B}{B}\right)^{0} \alpha^{2} .
$$

We hypothesize that this represents

$$
\frac{v_{p}^{(M)}}{\gamma_{a}^{(M)}}=(? ?)\left(\frac{L_{p}}{R_{w}}\right)^{2}\left(\frac{e N_{L}^{2}}{B}\right)^{1}\left(\frac{N^{\mathrm{tr}}}{N_{L}}\right)^{0} \alpha^{2},
$$

although $\gamma_{a}^{(M)}$ is only conceptual at present, since no magnetic mode has been observed.

The two "damping" processes are generically similar, as seen by comparing

$$
\gamma_{a}^{(M)}\left(T, B, n, L_{p}, R_{p}, \frac{\delta B}{B}\right) \text { vs } \gamma_{a}^{(E)}\left(T, B, n, R_{p}, L_{p}, V_{\mathrm{sq}}\right)
$$




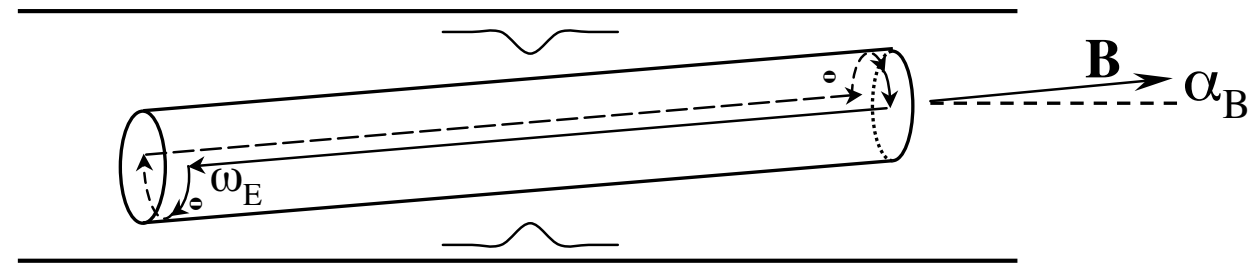

FIGURE 8. A tilted equliibrium has DC equilibrium currents which will be dissipated by a central squeeze.

The abrupt $T$ dependence of Fig. 2 and the $B^{-0.5}$ to $B^{-1}$ scaling of Eq. (1) probably apply to $\gamma_{a}^{(M)}$ also, since their nature is defined by the same process of scattering across a separatrix. Most striking as a difference is that the magnetic separatrix transport effects are independent of $\delta B / B$, down to our minimum of $\beta=10^{-3}$. Experimentally, this means that adding an external magnet to increase the mirror peak in Fig. 6 does not increase the asymmetry-induced transport. This surprising result is conceptually reasonable, in that the entire Maxwellian distribution of particles participates in magnetic separatrix crossings, no matter how small the separatrix angle $\beta^{1 / 2}$. Presumably, this process ceases only when the collisional (or non-linear) separatrix width [5] becomes comparable to the trapping width.

An alternate view of these TMP processes emphasizes the DC currents which must exist in tilted equilibria, as sketched in Fig. 8. The electron density at top-right is high, because it is close to the wall image charges. As these electrons $\mathbf{E} \times \mathbf{B}$ rotate, they flow axially down the front of the column, to form a high density at lower-left; they then reverse their axial flow along the back of the column. This gives zero net axial current of passing particles unless there is also a diocotron mode displacement of the entire column, in which case there are "sloshing currents" at frequency $f_{d}$ which are readily detected [10].

Dissipation of these asymmetry-induced currents through collisional scattering across electric and/or magnetic separatrices, at a large rate characterized by $\sqrt{v / f_{E}^{*}}$, is the essence of the TPM mode damping and transport, as illustrated in Fig. 9.

\section{POSSIBLE EXAMPLES}

It appears likely that TPM effects are pervasive, for two reasons: the rate is enhanced in low collisionality plasmas by $\sqrt{v / f_{E}^{*}}$, and magnetic trapping can be important even for $\delta B / B \sim 10^{-3}$. Thus, we suggest that TPM effects may be dominant in a variety of experimental situations.

The oft-observed (and oft-violated) $L^{2} / B^{2}$ scaling [6] for "anomalous" background transport probably results from magnetic asymmetries acting on magnetically trapped populations in moderate ridigity plasmas. The most direct demonstration of this is the $5 \times$ reduction obtained on CamV by removing the $\beta=10^{-3}$ mirror point. The EV apparatus 


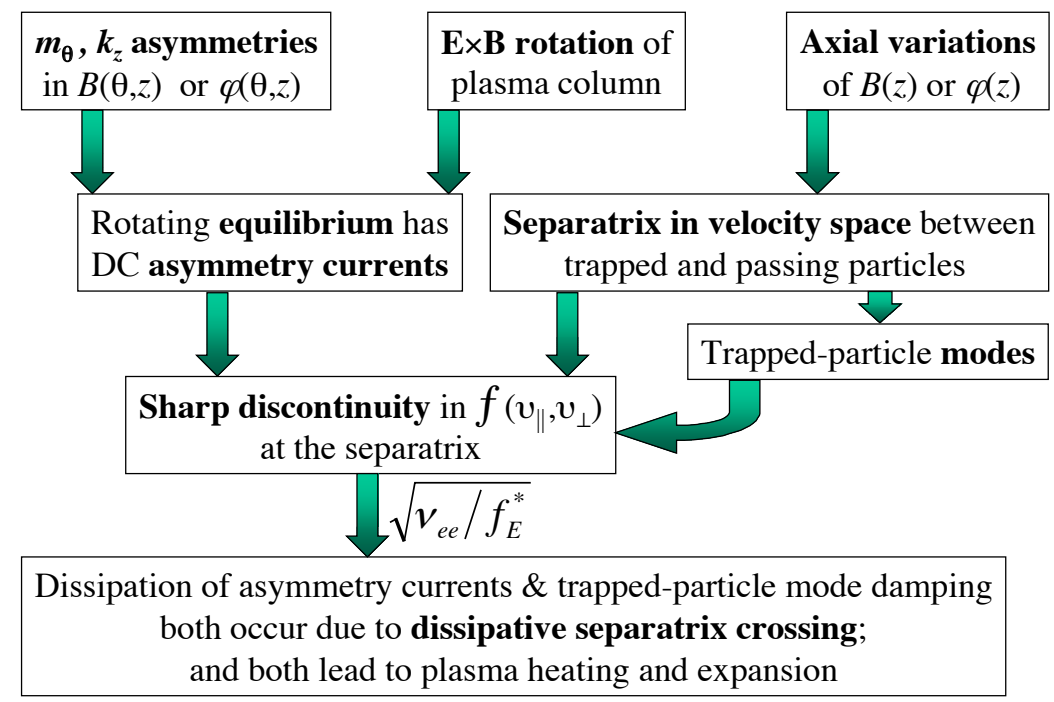

FIGURE 9. Conceptual outline of TPM effects.

has a gentle (axially extended) magnetic peak of $0.5 \%$.

More quantitative comparison requires knowledge of the trapping and of the background asymmetries. Multiple, or off-center, or extended trapping barriers presumably give rise to couplings to asymmetries with $(L / \pi) k_{z}=2,3, \ldots$, and this will change the scalings of Eq. (8).

Figure 10 presents an overview of electron and ion "background" expansion rates (scaled by $\sqrt{M_{i} / m_{e}}$ ), plotted versus the "rigidity" $\mathscr{R} \equiv f_{b} / f_{E}$. The original electron data from the $\mathrm{V}^{\prime}$ and $\mathrm{EV}$ apparatuses at $n \sim 10^{7} \mathrm{~cm}^{-3}$ and $T \sim 1 \mathrm{eV}$ gave the dashed

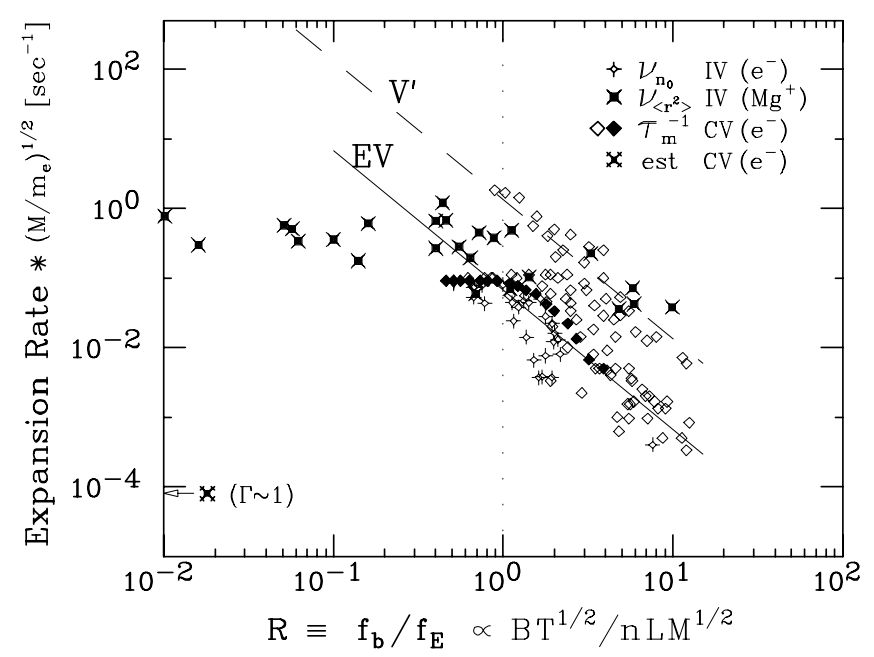

FIGURE 10. Scaled "background" loss rates versus rigidity for electrons and ions on 4 apparatuses. 
and solid lines, showing the puzzling $(L / B)^{2}$ scaling over about 4 decades. The higher density, warm electron data from IV was generically similar, but the floppy ions on IV show little correspondence with $\mathscr{R}^{-2}$. Electrons in CV at $n \sim 10^{8}$ look like a "swarm of killer bees," although individual temperature sequences often show an abrupt drop-off with temperature (e.g. solid diamonds). This abrupt temperature dependence is probably analogous to that of $\gamma_{a}^{(E)}$ in Fig. 2.

Neither "rigidity" nor "trapped particles" are valid terms for the $\mathscr{R} \ll 1$ ion points and the venerable Sendai [20] electron point $\left(n \sim 2 \times 10^{10}, T \sim 6^{\circ} \mathrm{K}, \mathscr{R} \sim 10^{-4}, \Gamma \sim 1\right)$. For the Sendai point the collisionality is exceedingly high: we obtain $v=3 \times 10^{10}$, compared to $f_{b} \sim 3 \times 10^{5}$ and $f_{E} \sim 5 \times 10^{8}$. Clearly no single process applies to all of Fig. 10 .

Nevertheless, the scalings of Eqs. (5-8) show striking correspondence to many prior results on asymmetry-induced transport. For magnetic field dependence, we expect $v_{p} \propto \gamma_{a}(B) B^{-1} \propto\left(B^{-1.5}\right.$ to $\left.B^{-2}\right)$, using the $\gamma_{a}(B)$ scaling of electric trapping. The observed length dependence of $v_{p}$ varies from $v_{p} \propto L_{p}^{2} N_{L}^{2}$ for fixed magnetic tilt $\alpha_{B}$ to $v_{p} \propto L_{p}^{-2} N_{L}^{0}$ for electric asymmetries, since fixed $V_{a}$ and fixed $L_{a}$ give $\alpha_{E} \propto L_{p}^{-2}$ in Eq. (3).

The abrupt decrease in transport observed for $\mathscr{R} \geq 10$ in recent experiments [8] is probably due to the temperature dependence of separatrix effects, as in Fig. 2. More importantly, $\mathscr{R}$ is not a globally relevant scaling parameter for transport due to trappedparticle separatrix crossings, since unperturbed particle parameters such as $f_{b}$ and $f_{E}$ cannot describe the nonlinearities of trapped orbits and non-Maxwellian separatrix velocity distributions.

Some of the most precise measurements of transport have been reported by Eggleston [9], using applied asymmetries which vary accurately as $\sin (\theta) \sin (n \pi z / L) \sin (2 \pi f t)$. Here, resonant particles are thought to be important, but the data does not match the theory of simple collisional scattering out of resonance. It appears likely that resonances are occurring in magnetically trapped particles with $\mathrm{v}_{z} \sim \beta^{1 / 2} \overline{\mathrm{v}} \sim .07 \overline{\mathrm{v}}$, and that collisions cause trapped/untrapped transitions, generating stronger transport. Here, experimental enhancement of separatrix crossings (as in Fig. 7) may help identify TPM effects.

Trapped-particle-mediated effects may also be occurring in recent experiments on transport from applied quadrupole magnetic asymmetries [10]. Here, a resonance is observed with $\mathrm{v}_{z}^{2}$ being 5 (or more) times less than $\overline{\mathrm{v}}^{2}$. This may possibly represent a " $\beta=1 / 5$ " reduction in $\mathrm{v}_{z}^{2}$; but the trapping characteristics of this system would be substantially more complex than any considered here.

Neutral collisions often give puzzling effects, including recent observations [21] of $v_{p} \propto B^{-1.5}$. Here, we note that $e-N$ collisions also contribute to separatrix crossings, so one would expect to observe expansion at a rate

$$
v_{p} \propto \sqrt{\frac{v_{\mathrm{ee}}+v_{\mathrm{eN}}}{f_{E}^{*}}} \approx \sqrt{\frac{v_{\mathrm{ee}}}{f_{E}^{*}}}\left(1+\frac{1}{2} \frac{v_{\mathrm{eN}}}{v_{\mathrm{ee}}}\right) .
$$

That is, TPM transport scalings of $B^{1.5}$ may be observed, even though $v_{p}$ increases linearly with pressure (with an offset).

Finally, we note that TPM effects cause strong exponential damping of the nominally stable diocotron modes (frequency $f_{m}$, damping $\gamma_{m}$, with $k_{z}=0, m_{\theta}=1,2 \ldots$ ), when 
$\theta$-asymmetries are also present. This may be viewed as collisional dissipation of the asymmetry- plus diocotron-induced sloshing currents discussed above. We find that this damping [22] scales as $\gamma_{m} / f_{m} \propto v_{p} \alpha^{2}$. Combined with $v_{p} \propto \sqrt{v / f} B^{-2} N_{L}^{2} \alpha^{2}$ from Eq. (5) and $f_{m} \propto N_{L} B^{-1}$, this implies $\gamma_{m} \propto \sqrt{v / f} B^{-3} N_{L}^{3} \alpha^{4}$. This $B^{-3}$ damping would be expected to dominate in experiments at low magnetic fields [21]. Moreover, for the dominant electrostatic asymmetry presumed in Ref. [11], Eq. (3) gives $\gamma_{m} \propto$ $\sqrt{v / f} B^{-3} N_{L}^{-1} V_{a}^{4}$; and this $N_{L}^{-1}$ scaling was indeed observed.

Targeted experiments incorporating separatrix manipulation and diagnostic techniques will be required to clarify the role of TPM transport and damping over the wide range of plasma parameters, trapping geometries, and asymmetry types in current experiments. Hopefully, this will combine with emerging theory to give a broader picture of TPM effects.

\section{ACKNOWLEDGMENTS}

This work is supported by National Science Foundation grant NSF-PHY9876999 and Office of Naval Research Grant N00014-96-1-0239.

\section{REFERENCES}

1. A.A. Kabantsev, C.F. Driscoll, T.J. Hilsabeck. T.M. O’Neil, and J.H. Yu, Phys. Rev. Lett. 87, 225002 (2001).

2. A.A. Kabantsev, J.H. Yu, R.B. Lynch, and C.F. Driscoll, Phys. Plasmas 10, 1628 (2003).

3. A.A. Kabantsev and C.F. Driscoll, Phys. Rev. Lett. 89, 245001 (2002).

4. A.A. Kabantsev and C.F. Driscoll, Rev. Sci. Instrum. 74, 1925 (2003).

5. T.J. Hilsabeck, A.A. Kabantsev, C.F. Driscoll, and T.M. O’Neil, Phys. Rev. Lett. 90, 245002 (2003).

6. C.F. Driscoll, K.S. Fine, and J.H. Malmberg, Phys. Fluids 29, 2015 (1986).

7. K.S. Fine, UCSD Ph.D. dissertation (1988).

8. J.M. Kriesel and C.F. Driscoll, Phys. Rev. Lett. 85, 2510 (2000).

9. D.L. Eggleston and B. Carillo, Phys. Plasmas 10, 1308 (2003).

10. E. Gilson and J. Fajans, Phys. Rev. Lett. 90, 015001 (2003).

11. E. Sarid, E. Gilson, and J. Fajans, Phys. Rev.Lett. 89, 105002 (2002).

12. S.F. Paul, et al., " $m=1$ Diocotron Mode Damping in the Electron Diffusion Gauge (EDG) Experiment," in Non-Neutral Plasma Physics IV, AIP Conf. Proc. 606, (F. Anderegg et al., eds.), 305 (2002).

13. G.W. Mason, Phys. Plasmas 10, 1231 (2003).

14. M.N. Rosenbluth, D.W. Ross, and D.P. Kostomarov, Nucl. Fusion 12, 3 (1972).

15. X.-P. Huang, F. Anderegg, E.M. Hollmann, T.M. O’Neil, and C.F. Driscoll, Phys. Rev. Lett. 78, 875 (1997).

16. F. Anderegg, E.M. Hollmann, and C.F. Driscoll, Phys. Rev. Lett. 81, 4875 (1998).

17. J.M. Kriesel, UCSD Ph.D. dissertation (1999).

18. J. Fajans, Phys. Plasmas 10, 1209 (2003).

19. G.W. Hart, Phys. Fluids B 3, 2987 (1991).

20. J.H. Malmberg et al., "The Cryogenic Pure Electron Plasma," in Proc. of 1984 Sendai Symposium on Plasma Nonlinear Phenomena (N. Sato, ed.), 31 (1984).

21. E.H. Chao et al., Phys. Plasmas 7, 831 (2000).

22. A.A. Kabantsev, to be published. 